\title{
A Weaving Rip-Stop Technique Leads to a Significantly Increased Load to Failure and Reduction in Suture-Tendon Cut-Through in a Biomechanical Model of Rotator Cuff Repair
}

\author{
Ryan A. Neeley, D.O., Miguel A. Diaz, M.S., R. Allen Gorman II, M.D., \\ Mark A. Frankle, M.D., and Mark A. Mighell, M.D.
}

Purpose: To present an alternative arthroscopic rip-stop technique with a single suture tape weaved through the tendon from anterior to posterior and to biomechanically test its strength against a control technique consisting of a single-row repair with simple sutures. Methods: This was a controlled biomechanical study. Dissection and harvesting of the supraspinatus muscle-tendon unit were performed along the cable in 9 matched-pair cadaveric shoulders. Samples were divided into 2 groups: simple suture repair only (SSR) and simple suture repair with rip-stop (SSPR). Biomechanical testing was performed with an initial preload, followed by cyclic loading and then ramp to failure. Peak-to-peak displacement, stiffness (in newtons per millimeter), load at failure (in newtons), and failure mechanism were recorded. Data were compared using the paired-sample $t$ test. Results: The average peak-to-peak displacement for SSR samples was not significantly different from that of SSPR samples $(P=.96)$. Similarly, elongation in the SSR and SSPR groups was not significantly different $(P=.82)$. Stiffness was significantly different between the SSR and SSPR groups $(P=.0054)$ : SSR samples were less stiff than SSPR samples. Moreover, SSR samples failed at significantly lower forces than did SSPR samples $(P=.028)$. A larger percentage of failures occurred due to tendon cut-through among SSR samples versus suture breakage among SSPR samples. Conclusions: An alternative rip-stop technique is presented in this biomechanical model that may assist surgeons to better deal with difficult rotator cuff repairs. Weaving a suture tape as a rip-stop can increase stiffness, achieve higher failure loads when compared with simple suture repair with no rip-stop, and reduce tendon cutthrough. Clinical Relevance: This study provides insight into a variation of rip-stop stitch techniques that may help solve the clinical problem of failure occurring at the suture-tendon interface, specifically tendon cut-through.

From Florida Orthopaedic Institute, Tampa, Florida, U.S.A. (R.A.N., M.A.F., M.A.M.); and Foundation for Orthopaedic Research $\theta$ Education, Tampa, Florida, U.S.A. (M.A.D., R.A.G.).

The authors report the following potential conflicts of interest or sources of funding: R.A.N. reports that this study was supported by a research grant provided by DePuy Mitek paid directly to the Foundation for Orthopaedic Research $\theta$ Education (FORE). This study was investigator initiated. M.A.D. reports that this study was supported by a research grant provided by DePuy Mitek paid directly to the institution (FORE). This study was investigator initiated. In addition, M.A.D. reports that the institution (FORE) has received a research grant from Zimmer Biomet, outside the submitted work. R.A.G. reports that this study was supported by a research grant provided by DePuy Mitek paid directly to the institution (FORE). This study was investigator initiated. M.A.F. reports that this study was supported by a research grant provided by DePuy Mitek paid directly to the institution (FORE). This study was investigator initiated. In addition, M.A.F. receives royalties from DJO Surgical, performs consulting for DJO Surgical, and receives speaking and educational engagement fees from DJO Surgical, outside the submitted work. M.A.M. reports that this study was supported by a research grant provided by DePuy Mitek paid directly to the institution (FORE). This study was investigator initiated. In addition, M.A.M. performs consulting for DePuy Synthes, DJO Surgical, and Stryker; receives speaking and educational engagement fees from DePuy Synthes, DJO Surgical, and Stryker; and receives royalties from DJO Surgical and NewClip Technics, outside the submitted work. Full ICMJE author disclosure forms are available for this article online, as supplementary material.

This research was performed at the Phillip Spiegel Orthopaedic Research Laboratory, Foundation for Orthopaedic Research and Education, Tampa, Florida, U.S.A.

This study was presented at the Fifth Annual Technology and Innovation in Orthopedics Symposium, hosted by the Holy Cross Orthopedic Institute, Fort Lauderdale, Florida, January 24th, 2020, and was the winner of the Best Fellow Abstract Presentation.

Received September 8, 2020; accepted April 24, 2021.

Address correspondence to Mark A. Mighell, M.D., Florida Orthopaedic Institute, $13020 \mathrm{~N}$ Telecom Pkwy, Tampa, FL 33637, U.S.A. E-mail: mmighell@floridaortho.com

(C) 2022 THE AUTHORS. Published by Elsevier Inc. on behalf of the Arthroscopy Association of North America. This is an open access article under the CC BY-NC-ND license (http://creativecommons.org/licenses/by-nc-nd/4.0/). 2666-061X/201538

https://doi.org/10.1016/j.asmr.2021.04.005 
$\mathbf{R}$ otator cuff repair continues to be one of the most commonly performed orthopaedic procedures, ${ }^{1-5}$ with over 460,000 surgical procedures per year in the United States. ${ }^{6,7}$ It has a long history of good clinical outcomes, but healing rates continue to be suboptimal in certain populations. ${ }^{8}$ Most rotator cuff repairs are performed in individuals older than 60 years, which unfortunately has been shown to be an independent risk factor for poor healing. ${ }^{9}$ Boileau et al. ${ }^{10}$ showed that after single-row arthroscopic repair, the rate of healing in patients older than 65 years was $43 \%$ compared with $95 \%$ in individuals younger than 55 years. Poor tissue quality is another independent risk factor for low healing rates after arthroscopic rotator cuff repair. ${ }^{11}$ This problem is encountered in approximately one-third of arthroscopic repairs and has been associated with a 3-fold increase in the risk of recurrent tear after repair. ${ }^{12}$

The transition of many rotator cuff repairs from using transosseous bone tunnels to using suture anchors has shifted the weak link of the repair from the bone to the tendon, ${ }^{13,14}$ with the common mode of failure seen now occurring at the suture-tendon interface. ${ }^{15-17}$ Research has been focused on exploring alternative suture techniques, materials (polyester vs polyblend), and configurations to increase the strength of the suture-tendon interface. ${ }^{14,18,19}$ One of the earliest suture techniques developed was the Mason-Allen stitch, which was later modified by Gerber et al. ${ }^{13,18}$ The purpose of these suture configurations-and the hope for any suture configuration-is to provide high initial fixation strength, minimize gap formation, and maintain mechanical stability until osteo-fibroblastic integration can occur. ${ }^{13,18}$ However, suture techniques such as the Mason-Allen and modified Mason-Allen stitch have proved to be difficult to perform in an arthroscopic manner and may lose loop security under a medial tensile load. ${ }^{20}$

A rip-stop stitch theoretically reduces the probability of suture cutout and maintains loop security. ${ }^{21}$ It consists of passing a horizontal mattress suture and then using a separate simple suture passed medially. ${ }^{22}$ Denard and Burkhart ${ }^{20,22}$ described using suture tape and double-row repair as a load-sharing rip-stop (LSRS) fixation construct, and Burkhart et al. ${ }^{11}$ were the first authors to provide a biomechanical validation. In this biomechanical study, Burkhart et al. ${ }^{11}$ compared the LSRS construct with a standard single-row repair construct. They showed that there was no difference in displacement between the 2 constructs but the ultimate load of the rip-stop construct was much higher.

Although studies involving different types of rip-stop configurations have provided promising results, there are still many variables and considerations to be addressed. Few biomechanical studies have evaluated the use of a rip-top stitch and its variations for rotator cuff repair in human cadaveric models. To date, biomechanical studies that have evaluated alternative techniques have included the studies of Burkhart et al., ${ }^{11}$ assessing the LSRS construct; Meisel et al., ${ }^{23}$ performing linked single-row repair; and Noyes et al., ${ }^{24}$ using a knotless rip-stop construct. In each of the referenced studies, the use of cadaveric bone and bone anchors introduced a confounding variable when evaluating alternative rip-stop techniques. Although anchor pullout is a clinically relevant mode of construct failure and presentation of such data is important, the question concerning an alternative approach for poor tendon quality remains. By eliminating the use of bone anchors, this study design allows a focus on the tendonsuture interface.

The purpose of this biomechanical study was to present an alternative arthroscopic rip-stop technique with a single suture tape weaved through the tendon from anterior to posterior and to test its strength against a control technique consisting of a single-row repair with simple sutures. We hypothesized that when compared with the simple suture repair, the suture tape rip-stop repair would have a significantly increased load to failure and overall fewer failures occurring at the suture-tendon interface.

\section{Methods}

\section{Sample Preparation}

A total of 10 matched pairs $(\mathrm{N}=20)$ of cadaveric shoulders $(7$ male and 3 female cadavers; average weight, $78.3 \mathrm{~kg}$; age range, 65-90 years [mean age, 77 years]), obtained from a United Tissue Network tissue bank, were used for this study and maintained frozen at $-20^{\circ} \mathrm{C}$ until the day prior to instrumentation. Specimens were thawed overnight at room temperature for dissection and harvesting of the supraspinatus muscletendon unit (MTU). The MTU samples were harvested by bordering the rotator cable and included only the longitudinal fibers. All 20 MTUs were visually inspected by a fellowship-trained surgeon (R.A.N.). Donors were excluded if they had gross abnormalities, injuries, or previous repairs. After the inspection, it was concluded that 1 matched pair had to be removed because of abnormalities (cyst formation). This was a time-zero, controlled biomechanical study in which the remaining donor sets were randomly divided into the following testing groups such that each pair received one of each repair construct (Fig 1): control group (simple suture repair only [SSR], 9 supraspinatus specimens) and experimental group (simple suture repair with PermaTape [DePuy Mitek] [SSPR], 9 
supraspinatus specimens). The control group consisted of 2 simple sutures (No. 2 Orthocord; DePuy Mitek).

The experimental group incorporated simple suture repair augmented with PermaTape (suture tape). This technique uses a single suture tape that is weaved into the tendon with multiple passes from anterior to posterior as the rip-stop stitch. The suture loop and free end of suture tape were secured around a cylinder that was rigidly fixed to the base of the testing machine. Independently, a single-row repair was performed with a simple stitch passed medially to the ripstop stitch (Fig 2). This was tested against a control technique consisting of a single-row repair with simple sutures.

Digital calipers were used to measure the distance from the edge of the tendon to the entry site of the suture, as well as the thickness and width of the tendon. ${ }^{13,18}$ Specimens in the SSR group received the appropriate stitch configuration about the central line of axis (measured as half the length of the width) 1 $\mathrm{cm}$ from the lateral end of the tendon. Specimens in the SSPR group were augmented with suture tape, which was achieved by weaving (running) a continuous line of suture tape in a horizontal fashion. The 2 simple sutures were then passed in a similar fashion to those in the SSR group, $5 \mathrm{~mm}$ medial to the suture tape passes into the tendon (Fig 3). Both the suture and suture tape ends were secured around a fixed cylinder $1 \mathrm{~cm}$ in diameter. ${ }^{14}$ The sutures were secured to the cylinder with 1 surgeon's knot, followed by 5 alternating half-hitches, such that each group had 2 points of contact around the post. Clinically, these free ends would be secured with an anchor.

The final length of suture used in each specimen was $4 \mathrm{~cm}$ (from the insertion point to the knot around the post). To ensure equal length and tension were maintained during repair, predetermined regions (used for alignment) were colored onto the suture and suture tape with a skin marker, prior to repair. The aforementioned fellowship-trained surgeon (R.A.N.) performed all repairs in an open setting using arthroscopic knot-tying techniques and instrumentation. An Expressew suture passer (DePuy Mitek) was used to pass suture and suture tape through the tendon. Throughout instrumentation and testing, samples were kept hydrated with $0.9 \%$ saline solution.

\section{Biomechanical Testing}

Biomechanical evaluation followed a testing protocol similar to protocols well documented in the published literature. ${ }^{11,13-15,22,25,26}$ Cyclic tensile testing was performed on a servo-hydraulic testing machine (MTS Bionix; MTS Systems, Eden Prairie,
MN) equipped with a 5-kN load cell. A standardized length of muscle belly of the supraspinatus, $4 \mathrm{~cm}$, was coupled to the MTS actuator by passing it through a cryo-clamp cooled by dry ice to a temperature of $-22^{\circ} \mathrm{C}$ (a critical temperature, monitored by a probe, shown to ensure proper coupling). ${ }^{27-29}$ This cryo-clamp allowed the tissue to be gripped (by compression of plates) and fixed (by freezing) to the plate surface, eliminating tissue slippage commonly associated with this testing environment (Fig 4).

The suture loop and free end of suture tape were secured around the cylinder that was rigidly fixed to the base of the MTS machine. The mechanical testing model was designed to test the suture-tendon junction and did not incorporate the variability introduced by using humeri or suture anchors (Fig 5). The length of suture loop, tendon grip length, and length of frozen tendon were standardized and measured across all specimens. Before testing, the tendon was inspected by visual and tactile means to ensure it was not frozen.

All testing samples were preconditioned to normalize viscoelastic effects and testing variability through application of a $5-\mathrm{N}$ preload for 2 minutes. The samples were then cyclically loaded from 5 to 30 $\mathrm{N}$ for 500 cycles at $0.25 \mathrm{~Hz}$. After cyclic testing, ramp to failure at $1 \mathrm{~mm} / \mathrm{s}$ was performed. During cyclic loading, peak-to-peak displacement data were collected from the actuator's linear variable differential transformer, defined as the average of maximum and minimum displacement across the last 3 cycles. During cyclic loading, a displacement distance of 5 $\mathrm{mm}$ or greater was considered to indicate failure. ${ }^{19,30}$ Elongation was defined as the difference in displacement (along y-axis) between the first cyclic peak and last cyclic peak (Fig 6A). During ramp to failure, stiffness (in newtons per millimeter), load at failure (in newtons), and failure mechanism were recorded. Stiffness was defined as the linear portion of the forceversus-displacement curve (Fig 6B). Failure was defined as the first significant decrease in the monotonically increasing force profile, at which the peak load at failure was recorded.

On the basis of previously published work of a similar scope, ${ }^{11,13,15,23}$ a large effect size (Cohen $d$ of 0.8 ) was used for an a priori power analysis. By use of a matched-pair experimental design with a 2-tailed hypothesis test with a significance threshold of .05 , the study was powered at the 0.80 level with 8 pairs of specimens ( $G^{*}$ Power, version 3.1.9.2; Franz Faul). Using 10 pairs of specimens allowed for any possible tissue rejection or unforeseen failures while retaining proper study power. 
A

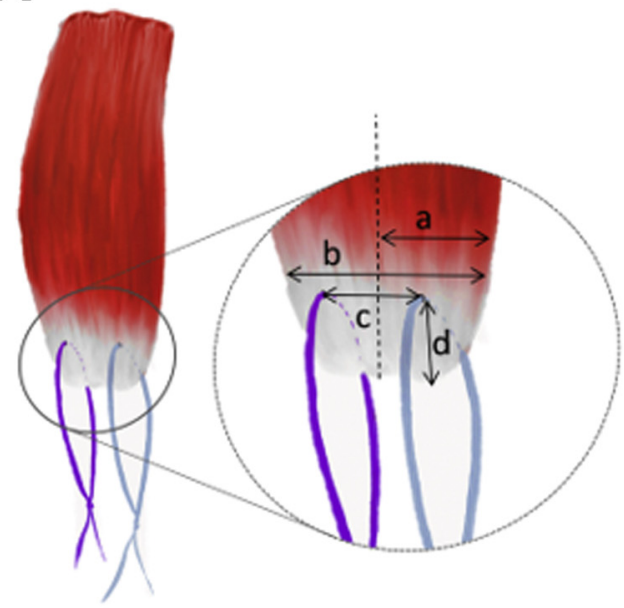

B

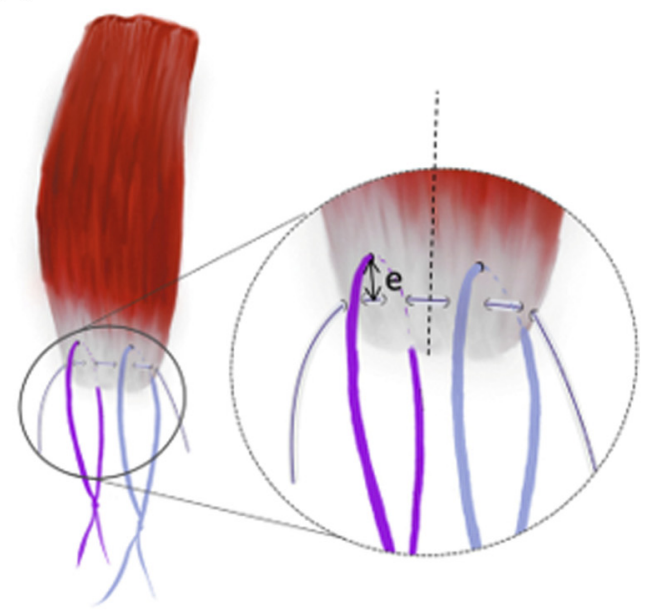

Fig 1. (A) Simple suture repair only. Distance a is calculated as b/2, in which b is the tendon width. Suture is passed $1 \mathrm{~cm}$ (distance d) from the distal end of the tendon, spaced $1.5 \mathrm{~cm}$ apart about the midline (distance c). (B) Simple suture repair with PermaTape. Similar standardization as for simple suture repair is followed, with the addition of suture tape placed $5 \mathrm{~mm}$ (distance e) from the suture passes.

\section{Statistical Analysis}

Paired-sample $t$ tests were performed on tendon measurements between test groups, and no statistically significant differences were found (Table 1).
Moreover, paired-sample $t$ tests were performed to identify differences in stiffness, ultimate failure load, peak-to-peak displacement, and elongation between the control and experimental groups. Data
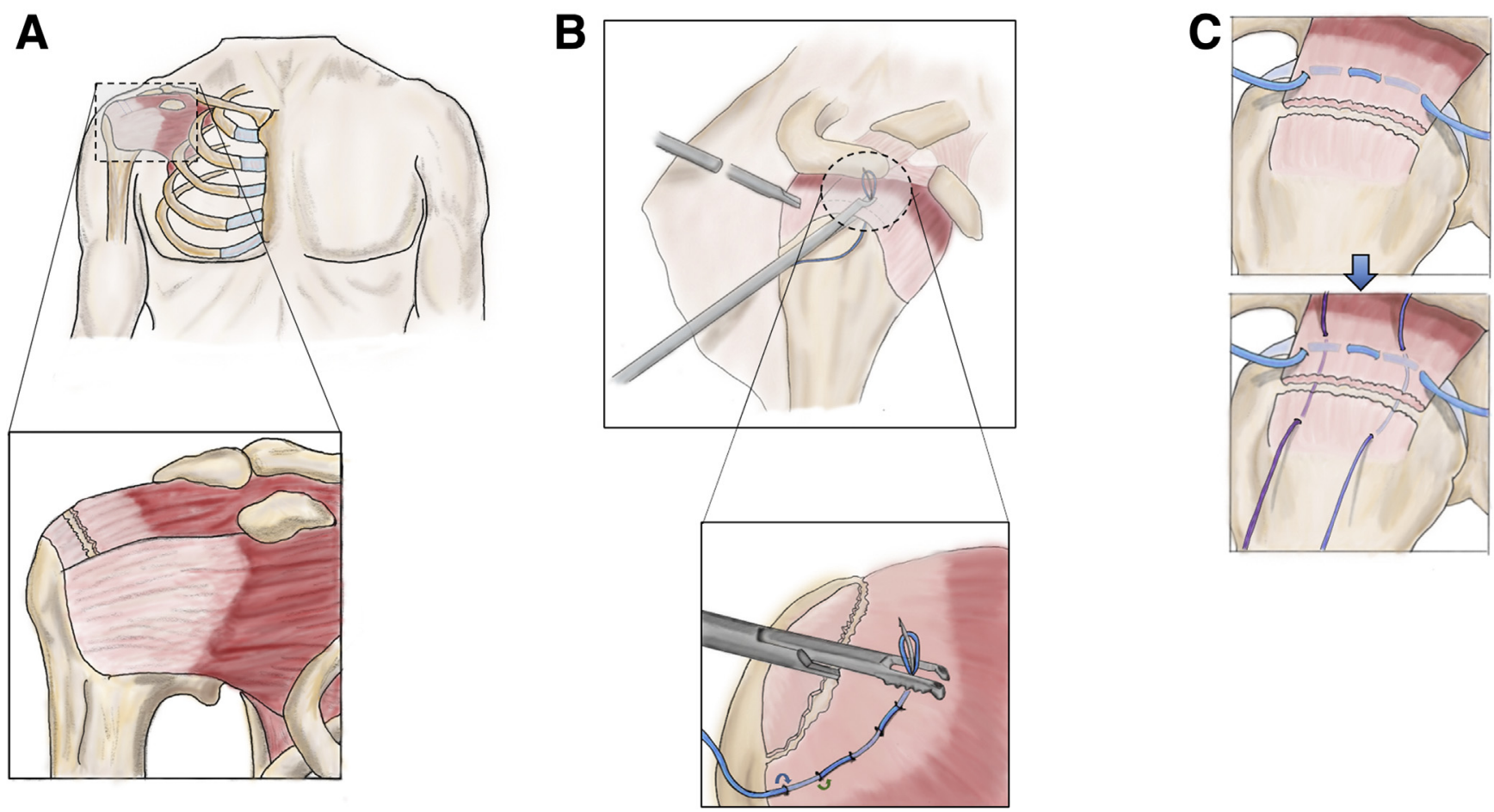

Fig 2. Right shoulder. (A) Anterior view of injured shoulder. (B) Suture tape weaved through tendon using arthroscopic instruments. (C) Arthroscopic view of shoulder from lateral portal. Suture tape is first passed through the tendon in a weaving fashion to act as a rip-stop, followed by the passage of simple sutures medial to the suture tape. 


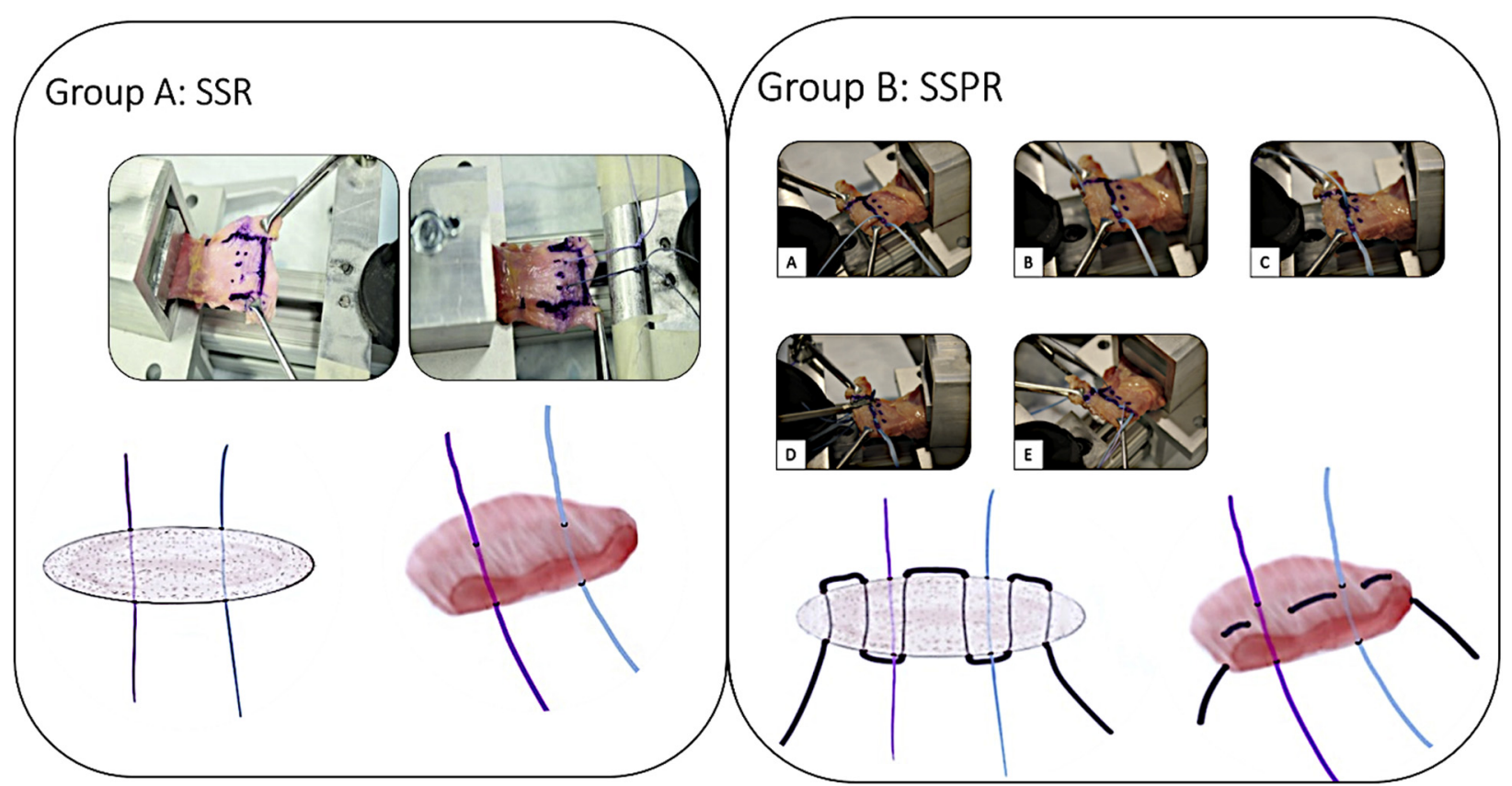

Fig 3. Instrumentation for suturing was facilitated using a custom clamping jig, allowing the tendon to be slightly tensioned. A skin marker was used to identify suture placement for both study groups. Suture passage is shown for samples with simple suture repair only (SSR), along with a cross-sectional view. Weaving of the suture tape in a step-by-step manner (A-E) is shown for samples with simple suture repair with PermaTape (SSPR), along with a cross-sectional view.

are presented as mean \pm standard deviation. All statistical comparisons were performed with SPSS software (version 22; IBM) at a significance level of $\alpha=.05$.

\section{Results}

The modes of failure were classified as either suture pull-through (tendon tear); suture breakage; or a combination of tendon tear through, shortly followed by suture breakage (Table 2). Stiffness was significantly different between the SSR and SSPR groups $(P=$ .0054): SSR samples were less stiff than SSPR samples $(38.7 \pm 13.7 \mathrm{~N} / \mathrm{mm}$ vs $50.0 \pm 12.8 \mathrm{~N} / \mathrm{mm})$. Moreover, SSR samples failed at significantly lower forces than did SSPR samples $(296.9 \pm 118.7 \mathrm{~N}$ vs $428.2 \pm 130.9 \mathrm{~N}, P=$ .028) (Table 3).

The average peak-to-peak displacement in SSR samples was not significantly different from that in SSPR samples $(0.29 \pm 0.17 \mathrm{~mm}$ vs $0.28 \pm 0.087 \mathrm{~mm}, P=$ .96). Similarly, elongation in the SSR and SSPR groups were not significantly different $(1.2 \pm 0.34 \mathrm{~mm}$ and 1.3 $\pm 0.35 \mathrm{~mm}$, respectively; $P=.82$ ) (Table 3 ).

\section{Discussion}

The results of this study confirm our hypothesis that weaving the rotator cuff tendon with suture tape to act as a rip-stop suture would lead to a significantly increased load to failure and reduction in suturetendon cut-through. In this study, we present an alternative rip-stop technique with biomechanical validation that may assist surgeons to deal with repairs in which the single row or double rows may fall short owing to insufficient tendon mobility. This rip-stop technique consists of weaving a suture tape through the rotator cuff tendon with multiple passes from

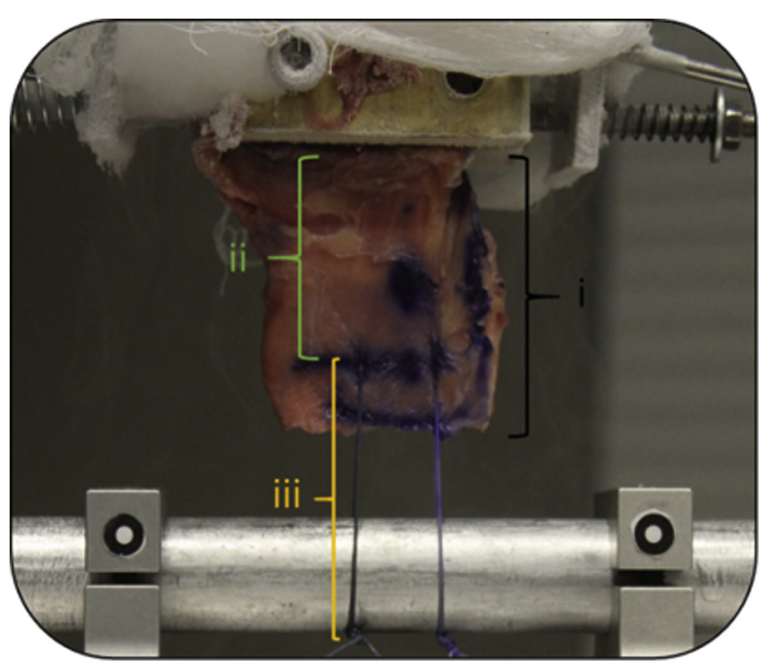

Fig 4. Standardization of tissue clamping showing the clamp distance of $4 \mathrm{~cm}$ from the tendon edge (i), the distance between the clamp and repair at $3 \mathrm{~cm}$ (ii), and the suture length of $4 \mathrm{~cm}$ (iii). 
A
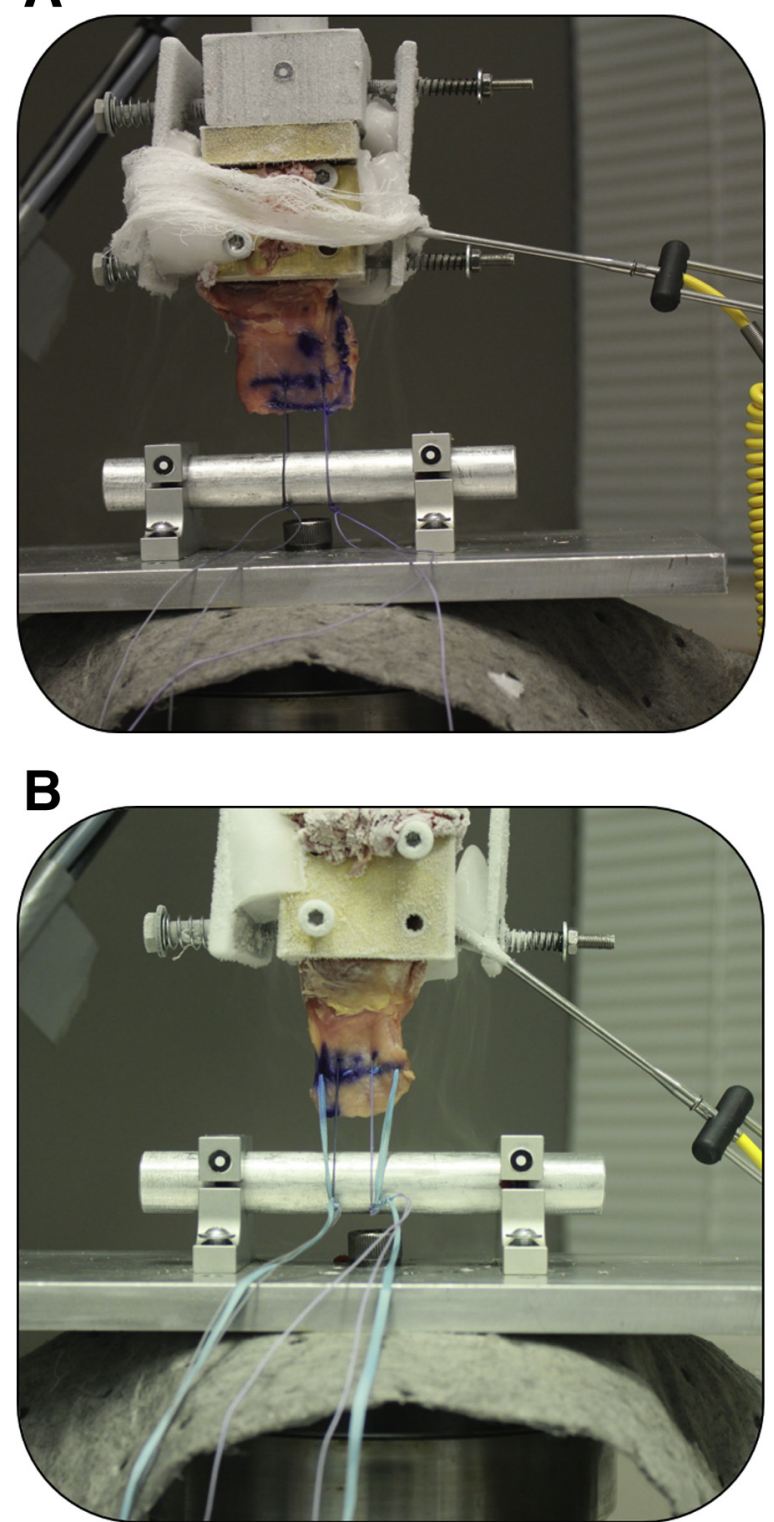

Fig 5. Samples undergoing simple suture repair only (SSR) (A) and simple suture repair with PermaTape (SSPR) (B) loaded on a testing frame in which dry ice was secured around the clamp. The temperature probe is inserted between the clamping surfaces within the tissue to monitor temperature decreases.

anterior to posterior and then passing simple sutures medial to the suture tape. The interaction at the suturetendon interface was evaluated without the added variables of humeri or suture anchors. This study was performed to determine whether this technique would provide the anticipated improvement in mechanical performance at the suture-tendon interface.

Clinically, suture cutout in rotator cuff tendons is often referred to as the "cheese-wiring" effect. ${ }^{31}$
Biomechanically, this can be captured as tendon elongation. Rip-stop configurations have been developed to increase fixation at the suture-tendon interface and should, in theory, decrease suture cutout. ${ }^{11,20}$ In our study, there was no significant difference between the control (SSR) group and experimental (SSPR) group regarding elongation within our cyclic loading protocol. Burkhart et al. ${ }^{11}$ observed similar results in their LSRS study and described their reasoning in great detail. We agree with their findings that it is not possible to fully engage the rip-stop suture until some displacement occurs.

As this displacement occurs, the rip-stop becomes engaged and adds rigidity, increasing the ultimate failure load as observed in this study. Therefore, to minimize any suture cutout, it may be best to insert the ripstop suture as closely to the preferred repair as possible. There was no significant difference in elongation within cyclic loading; however, there were significant differences in both stiffness and load to failure between groups. SSR samples were less stiff than SSPR samples: $38.7 \pm 13.7 \mathrm{~N} / \mathrm{mm}$ versus $50.0 \pm 12.8 \mathrm{~N} / \mathrm{mm}$. Moreover, SSR samples failed at significantly lower forces than did SSPR samples: $296.9 \pm 118.7 \mathrm{~N}$ and $428.2 \pm$ $130.9 \mathrm{~N}$. These findings equate to an ultimate load to failure 1.4 times greater than that in the SSR group and stiffness 1.3 times greater.

Furthermore, a considerable difference was seen when evaluating the modes of failure between groups. In the SSR group, $89 \%$ of specimens ( 8 of 9 ) failed by suture cutout through the tendon and $11 \%$ ( 1 of 9) failed owing to suture breakage, whereas in the SSPR group, only $33 \%$ of samples ( 3 of 9 ) failed by suture cut-through whereas $44 \%$ (4 of 9) failed by suture breakage and $22 \%$ ( 2 of 9 ) failed owing to tendon tearing followed by suture breakage. Although it is not surprising that most of the samples in the SSR group failed by suture cutout, it is significant to note that nearly $70 \%$ of the specimens in the SSPR group failed by some other means not related to suture cutout. One mode of failure observed only in the SSPR group was described as "tendon tearing followed by suture breakage," in which the rotator cuff tear propagated parallel to the suture tape until the suture ruptured. Although this could be a catastrophic failure clinically, it seems unlikely given that this mechanism of failure occurred at supraphysiological forces. ${ }^{32-34}$ Various simulations use forces between 40 and $200 \mathrm{~N}$ to actuate the supraspinatus during arm elevation. ${ }^{34-37}$ Although both constructs on average performed beyond clinical demand, failure loads in the SSR group ranged from 140 to $476 \mathrm{~N}$, whereas those in the SSPR group ranged from 274 to $609 \mathrm{~N}$. The failure properties of supraspinatus tendons with intact humeral insertions are estimated to be above $800 \mathrm{~N} .^{38,39}$ Clinically, this finding may be pertinent to repairing various tears with poorquality tissue in which suture cut-through failure is common. ${ }^{15,31}$ 
A
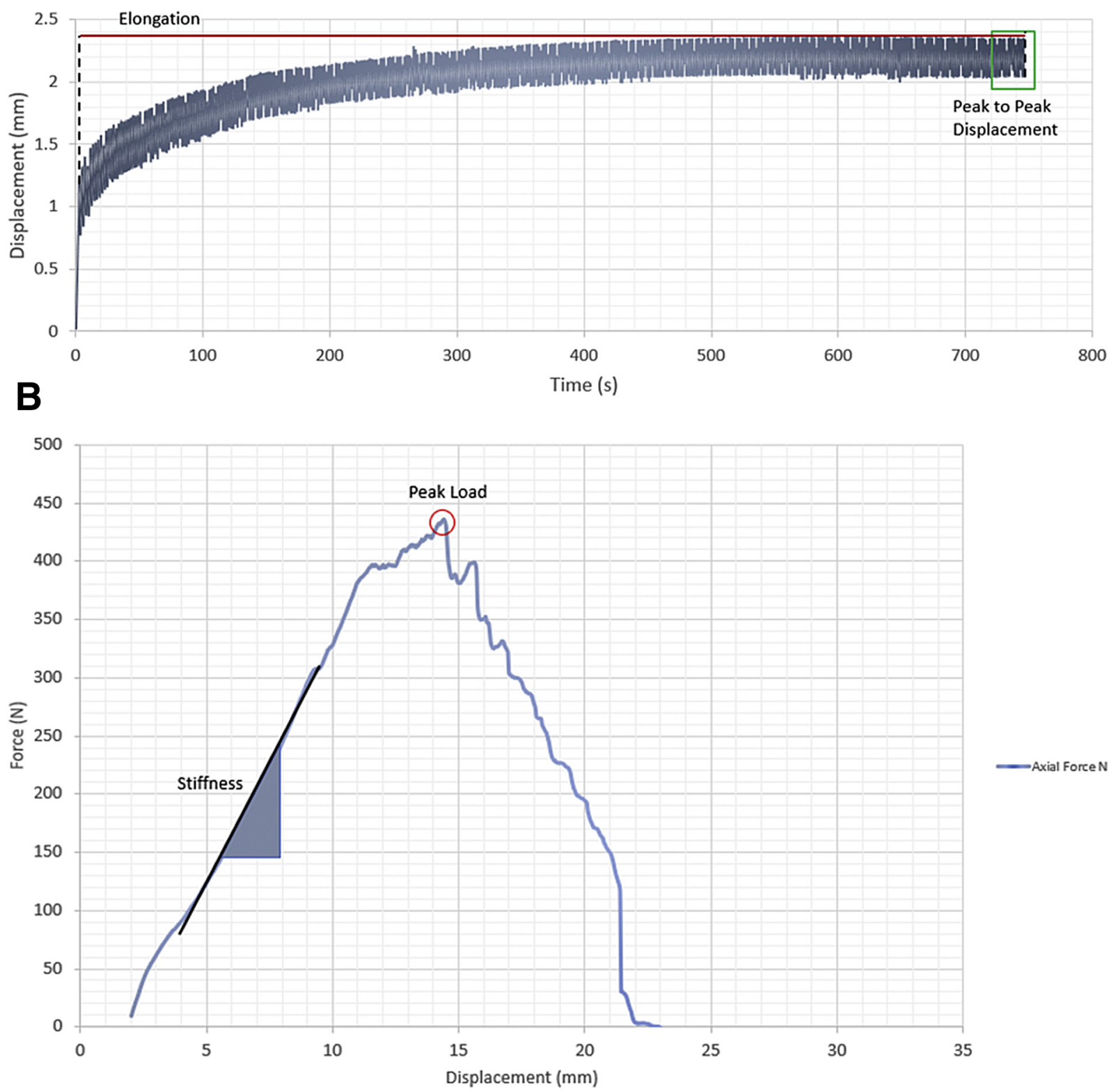

Fig 6. (A) Example of data output during cyclic loading, in which peak-to-peak displacement and elongation measurements are identified. (B) Stiffness and peak load information is gathered from the force-displacement curve created from data output during ramp to failure.

The results of this controlled biomechanical study provide evidence based on a cadaveric matched-pair model that adding a suture tape as a rip-stop can increase stiffness, achieve higher failure loads, and decrease failures at the suture-tendon interface when compared with simple suture repair with no rip-stop. Moreover, this study adds to the current body of literature and provides a promising variation of rip-stop stitch techniques that may help solve the clinical problem of failures occurring at the suture-tendon interface (tendon cut-through). The presented technique has been found to be easy to perform arthroscopically by the senior author (M.A.M.) in a limited group of patients with 2 tendon tears and tears with lateral tendon loss.

\section{Limitations}

This study is not without its limitations. This is a timezero, matched-pair cadaveric study that did not use anchors or humeri, and the results do not account for 
Table 1. Donor Demographic Characteristics and Tendon Measurements

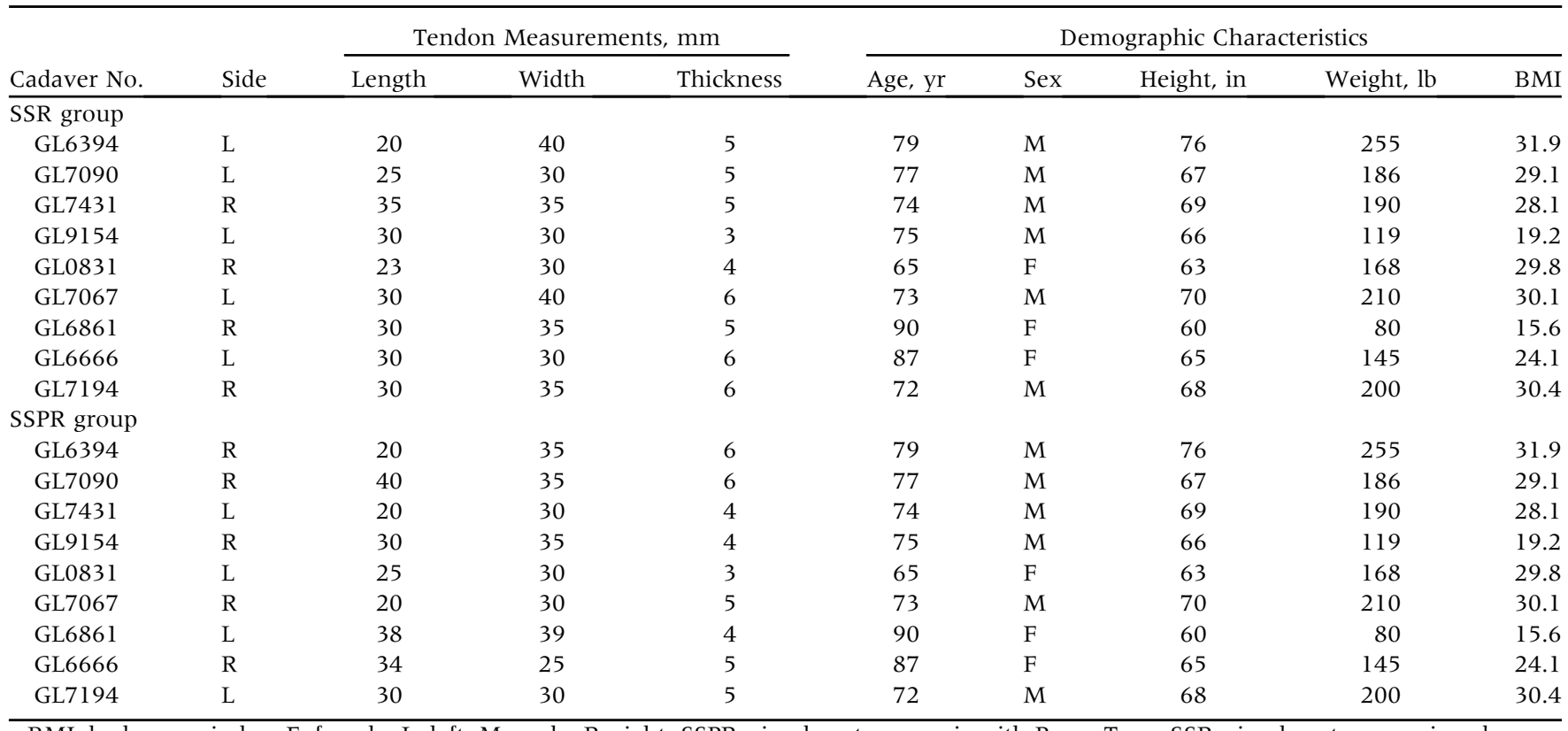

BMI, body mass index; F, female; L, left; M, male; R, right; SSPR, simple suture repair with PermaTape; SSR, simple suture repair only.

tissue healing that normally occurs in vivo. The average thickness of the tendons in this study measured over 3 $\mathrm{mm}$, which may not be the best representation of poor tendon quality but is comparable to that in other studies with similar experimental protocols. ${ }^{15,23,34}$ The use of matched cadaveric pairs minimizes the effect of tendon quality variation. The primary focus was the interaction at the suture-tendon interface, which was evaluated without the added variables of humeri or suture anchors because these can potentially add confounding factors to modes of failure. The placement of the ripstop was standardized at $5 \mathrm{~mm}$ from simple sutures; however, at this distance, the rip-stop may not have been engaged during cyclic testing. Knot security is another variable that may alter results; therefore, our procedure for knot tying and instrumentation was followed and performed by 1 surgeon to minimize technique variability using arthroscopic instruments. Our knot-tying technique consisted of 1 surgeon's knot and 5 alternating half-hitches and was not found to be a weak point in the construct because none of the failures occurred owing to knot failure. Finally, this study was performed in a non-aqueous environment; however, care was taken to continuously maintain tissue hydration with $0.9 \%$ saline solution.

\section{Conclusions}

An alternative rip-stop technique is presented in this biomechanical model that may assist surgeons to better deal with difficult rotator cuff repairs. Weaving a suture tape as a rip-stop can increase stiffness, achieve higher failure loads when compared with simple suture repair with no rip-stop, and reduce tendon cut-through.

Table 2. Failure Modes Observed During Ramp-to-Failure Testing in SSR and SSPR Groups

\begin{tabular}{lccc}
\hline & & Failure Mode, $\mathrm{n}(\%)$ & \\
\cline { 2 - 4 } Study Group & Suture Pull-Through & Suture Breakage & Tendon Tear Followed by \\
SSR & $8(89)$ & $1(11)$ & Suture Breakage \\
SSPR & $3(33)$ & $4(44)$ & $2(0)$ \\
\hline
\end{tabular}

SSPR, simple suture repair with PermaTape; SSR, simple suture repair only. 
References

1. Ensor KL, Kwon YW, DiBeneditto MR, Zuckerman JD, Rokito AS. The rising incidence of rotator cuff repairs. J Shoulder Elbow Surg 2013;22:1628-1632.

2. Monrad N, Ganestam A, Kallemose T, Barfod KW. Alarming increase in the registration of degenerative rotator cuff-related lesions a nationwide epidemiological study investigating 244,519 patients. Knee Surg Sports Traumatol Arthrosc 2018;26:188-194.

3. Paloneva J, Lepola V, Äärimaa V, Joukainen A, Ylinen J, Mattila VM. Increasing incidence of rotator cuff repairs-A nationwide registry study in Finland. BMC Musculoskelet Disord 2015;16:189.

4. White J, Titchener A, Fakis A, Tambe A, Hubbard R, Clark D. An epidemiological study of rotator cuff pathology using The Health Improvement Network database. Bone Joint J 2014;96:350-353.

5. Zhang AL, Montgomery SR, Ngo SS, Hame SL, Wang JC, Gamradt SC. Analysis of rotator cuff repair trends in a large private insurance population. Arthroscopy 2013;29: 623-629.

6. iData Research. Over 460,000 rotator cuff surgeries per year reported in the United States by iData Research. Published 2018, https://idataresearch.com/over-460000rotator-cuff-surgeries-per-year-reported-in-the-unitedstates-by-idata-research/. Accessed December 12, 2019.

7. Synthasome. Literature review: Effectiveness of rotator cuff surgery, http://www.synthasome.com/review-ofeffectiveness.php. Accessed December 12, 2019.

8. Petit CJ, Boswell R, Mahar A, Tasto J, Pedowitz RA. Biomechanical evaluation of a new technique for rotator cuff repair. Am J Sports Med 2003;31:849-853.

9. Bisson LJ, Manohar LM, Wilkins RD, Gurske-Deperio J, Ehrensberger MT. Influence of suture material on the biomechanical behavior of suture-tendon specimens: A controlled study in bovine rotator cuff. Am J Sports Med 2008;36:907-912.

10. Boileau P, Brassart N, Watkinson DJ, Carles M, Hatzidakis AM, Krishnan SG. Arthroscopic repair of fullthickness tears of the supraspinatus: Does the tendon really heal? J Bone Joint Surg Am 2005;87:1229-1240.

11. Burkhart SS, Denard PJ, Konicek J, Hanypsiak BT. Biomechanical validation of load-sharing rip-stop fixation for the repair of tissue-deficient rotator cuff tears. Am J Sports Med 2014;42:457-462.

12. Nho SJ, Brown BS, Lyman S, Adler RS, Altchek DW, MacGillivray JD. Prospective analysis of arthroscopic rotator cuff repair: Prognostic factors affecting clinical and ultrasound outcome. J Shoulder Elbow Surg 2009;18: 13-20.

13. Gerber C, Schneeberger AG, Beck M, Schlegel U. Mechanical strength of repairs of the rotator cuff. J Bone Joint Surg Br 1994;76:371-380.

14. Ma CB, MacGillivray JD, Clabeaux J, Lee S, Otis JC. Biomechanical evaluation of arthroscopic rotator cuff stitches. J Bone Joint Surg Am 2004;86:1211-1216.

15. Owens BD, Algeri J, Liang V, DeFroda S. Rotator cuff tendon tissue cut-through comparison between 2 hightensile strength sutures. J Shoulder Elbow Surg 2019;28: 1897-1902. 
16. Cummins CA, Murrell GA. Mode of failure for rotator cuff repair with suture anchors identified at revision surgery. J Shoulder Elbow Surg 2003;12:128-133.

17. Ladermann A, Denard PJ, Burkhart SS. Management of failed rotator cuff repair: A systematic review. J ISAKOS 2016;1:32-37.

18. Gerber C, Fuchs B, Hodler J. The results of repair of massive tears of the rotator cuff. J Bone Joint Surg Am 2000;82:505-515.

19. Schneeberger AG, von Roll A, Kalberer F, Jacob HA, Gerber C. Mechanical strength of arthroscopic rotator cuff repair techniques: An in vitro study. J Bone Joint Surg Am 2002;84:2152-2160.

20. Denard PJ, Burkhart SS. A load-sharing rip-stop fixation construct for arthroscopic rotator cuff repair. Arthrosc Tech 2012;1:e37-e42.

21. Castagna A, Garofalo R, Conti M, Borroni M, Snyder SJ. Arthroscopic rotator cuff repair using a triple-loaded suture anchor and a modified Mason-Allen technique (Alex stitch). Arthroscopy 2007;23:440.el-440.e4.

22. Denard PJ, Burkhart SS. Techniques for managing poor quality tissue and bone during arthroscopic rotator cuff repair. Arthroscopy 2011;27:1409-1421.

23. Meisel AF, Henninger HB, Barber FA, Getelman MH. Biomechanical comparison of standard and linked singlerow rotator cuff repairs in a human cadaver model. Arthroscopy 2017;33:938-944.

24. Noyes MP, Lederman E, Adams CR, Denard PJ. Tripleloaded suture anchors versus a knotless rip stop construct in a single-row rotator cuff repair model. Arthroscopy 2018;34:1414-1420.

25. Ma CB, Comerford L, Wilson J, Puttlitz CM. Biomechanical evaluation of arthroscopic rotator cuff repairs: Double-row compared with single-row fixation. J Bone Joint Surg Am 2006;88:403-410.

26. Bisson LJ, Manohar LM. A biomechanical comparison of the pullout strength of No. 2 FiberWire suture and 2-mm FiberWire tape in bovine rotator cuff tendons. Arthroscopy 2010;26:1463-1468.

27. Santoni BG, McGilvray KC, Lyons AS, et al. Biomechanical analysis of an ovine rotator cuff repair via porous patch augmentation in a chronic rupture model. Am J Sports Med 2010;38:679-686.

28. Scheibel MT, Habermeyer P. A modified Mason-Allen technique for rotator cuff repair using suture anchors. Arthroscopy 2003;19:330-333.
29. Barber FA, Drew OR. A biomechanical comparison of tendon-bone interface motion and cyclic loading between single-row, triple-loaded cuff repairs and double-row, suture-tape cuff repairs using biocomposite anchors. Arthroscopy 2012;28:1197-1205.

30. Burkhart SS, Pagan JLD, Wirth MA, Athanasiou K. Cyclic loading of anchor-based rotator cuff repairs: Confirmation of the tension overload phenomenon and comparison of suture anchor fixation with transosseous fixation. Arthroscopy 1997; 13:720-724.

31. Lambrechts M, Nazari B, Dini A, et al. Comparison of the cheese-wiring effects among three sutures used in rotator cuff repair. Int J Shoulder Surg 2014;8:81-85.

32. Leschinger T, Birgel S, Hackl M, Staat M, Müller LP, Wegmann K. A musculoskeletal shoulder simulation of moment arms and joint reaction forces after medialization of the supraspinatus footprint in rotator cuff repair. Comput Methods Biomech Biomed Engin 2019;22:595-604.

33. Gerber C, Snedeker JG, Baumgartner D, Viehöfer AF. Supraspinatus tendon load during abduction is dependent on the size of the critical shoulder angle: A biomechanical analysis. J Orthop Res 2014;32:952-957.

34. Aliaj K, Henninger HB, Tetreault-Paquin JE, Getelman MH, Donahue JP. Biomechanics of an interlinked suture anchor rotator cuff repair in a human cadaveric model. JSES Open Access 2019;3:70-76.

35. Giles JW, Ferreira LM, Athwal GS, Johnson JA. Development and performance evaluation of a multi-PID muscle loading driven in vitro active-motion shoulder simulator and application to assessing reverse total shoulder arthroplasty. J Biomech Eng 2014;136:121007.

36. Hansen ML, Otis JC, Johnson JS, Cordasco FA, Craig EV, Warren RF. Biomechanics of massive rotator cuff tears: Implications for treatment. J Bone Joint Surg Am 2008;90: 316-325.

37. Henninger HB, Barg A, Anderson AE, Bachus $\mathrm{KN}$, Tashjian RZ, Burks RT. Effect of deltoid tension and humeral version in reverse total shoulder arthroplasty: A biomechanical study. J Shoulder Elbow Surg 2012;21: 483-490.

38. Itoi E, Berglund LJ, Grabowski JJ, et al. Tensile properties of the supraspinatus tendon. J Orthop Res 1995;13: 578-584.

39. Matsuhashi T, Hooke AW, Zhao KD, et al. Tensile properties of a morphologically split supraspinatus tendon. Clin Anat 2014;27:702-706. 